\title{
On capacity of OFDMA-based IEEE802.16 WiMAX including Adaptive Modulation and Coding (AMC) and inter-cell interference
}

\author{
Chadi Tarhini, Tijani Chahed \\ GET/Institut National des Télécommunications - UMR CNRS 5157 \\ 9 rue C. Fourier - 91011 Evry CEDEX - France \\ \{chadi.tarhini, tijani.chahed\}@int-evry.fr
}

\begin{abstract}
We study in this paper the capacity of the downlink of OFDMA-based IEEE802.16 WiMAX system in the presence of two types of traffic, streaming and elastic. We focus in particular on the impact of Adaptive Modulation and Coding (AMC) as well as inter-cell interference resulting from different frequency reuse schemes. Several performance measures, namely blocking rates, mean transfer time and the mean number of collisions between two OFDMA WiMAX cells, are then derived and quantified. We show that reuse partitioning results in a lower blocking probability for streaming flows in the inner region but a much higher one for elastic flows in the outer region; the overall cell throughput increases meaning that the decrease in the number of collisions improves the overall throughput.
\end{abstract}

\section{INTRODUCTION}

WiMAX systems are based on versions $d$ and $e$ of the IEEE802.16 standard which defines a physical (PHY) and medium access control (MAC) layers for broadband wireless access systems operating at frequencies below $11 G H z$. The first of these standards, published in 2004, addresses fixed services while the second, published in 2005 , is intended for mobile services.

The IEEE 802.16e specifications define three different PHY layers: single-carrier transmission, Orthogonal FrequencyDivision Multiplexing (OFDM), and OFD Multiple Access (OFDMA). The multiple access technique used in the first two of these PHY specifications is pure TDMA, while the third technique uses both the time and frequency dimensions for resource allocation. From these three PHY technologies, OFDMA has been selected by WiMAX Forum [1] as the basic technology for portable and mobile applications. Compared to TDMA, OFDMA leads to a significant cell range extension. This is due to the fact that the transmit power of the mobile station is concentrated in a small portion of the channel bandwidth which increases the Signal-to-Noise Ratio (SNR) at the receiver. Another interesting feature of OFDMA is that it eases the deployment of networks with a frequency reuse factor of 1 , thus eliminating the need for frequency planning.

In wireless communication systems, random fluctuations prevent the continuous use of highly bandwidth-efficient modulation, and therefore Adaptive Modulation and Coding (AMC) has become a standard approach in recently developed wireless standards, including WiMAX. The idea behind AMC is to dynamically adapt the modulation and coding scheme to the channel conditions so as to achieve the highest spectral efficiency at all times [2]. Adaptive modulation changes the coding scheme and/or modulation method depending on channel-state information - choosing it in such a way that it squeezes the most out of what the channel can transmit. In OFDMA, modulation and/or coding can be chosen differently for each sub-carrier, and it can also change with time. Indeed, in the IEEE802.16 standard, coherent modulation schemes are used starting from low efficiency modulations (BPSK with coding rate $1 / 2$ ) to very high efficiency ones (64-QAM with coding rate 3/4) depending on the SNR. It has been shown that systems using adaptive modulation perform better than systems whose modulation and coding are fixed [3]. Adaptive modulation increases data transmission throughput and the system reliability by using different constellation size on different sub-carriers.

In a cellular OFDMA system, adjacent cells using subcarriers of exactly the same frequency and time can cause interference to one another unless inter-cell interference mitigation techniques are applied. This interference takes the form of collisions, the number of which increases as mobile stations get closer to the edge of the cell. To combat this, a reuse partitioning [4] scheme can be applied and controls the reuse factor in different parts of the cell.

In the literature, several works considered the capacity of broadband wireless OFDMA-based WiMAX systems. In Reference [10] for instance, work has been done on one region only without taking AMC into account. In Reference [11], focus was mostly on interference in a multi-cell setting without reuse partitioning. In this work, we focus on the capacity of OFDMA-based WiMAX systems considering both intra-cell AMC and inter-cell collisions in the presence of two types of traffic, constant-bit-rate streaming flows, such as voice, and elastic data ones which are governed by TCP at the transport layer, with and without reuse partitioning.

\section{PhySiCAL LAYER IN WIMAX}

\section{A. OFDMA sub-carrier allocations}

OFDMA is a multiple access technique which divides the total Fast Fourier Transform (FFT) space into a number of sub-channels (set of sub-carriers that are assigned for data exchange) whereas the time resource is divided into time slots 
(i.e. in WiMAX OFDMA PHY [3], the minimum frequencytime unit of sub-channelization is one slot, which is equivalent to 48 sub-carriers) and a frame is constructed by a number of slots. Let $N$ denote the total number of sub-carriers of all types, pilots, guard and data, (and which is equal to the size of the FFT) and let $N^{\prime}$ denote the total number of data subcarriers after reserving the pilot and guard sub-carriers which we divide into $L$ groups, each with $K=N^{\prime} / L$ data subcarriers.

In OFDMA-based WiMAX system, resource allocation is done in time-frequency domain: a call may share a sub-channel with other users. This is illustrated in Figure 1 where users $2,3,4$ and 5 occupy each one sub-channel half of the time while user 1 occupies one sub-channel all the time. With OFDMA, the user device could choose sub-channels based on geographical location with the potential of eliminating the impact of deep fades.

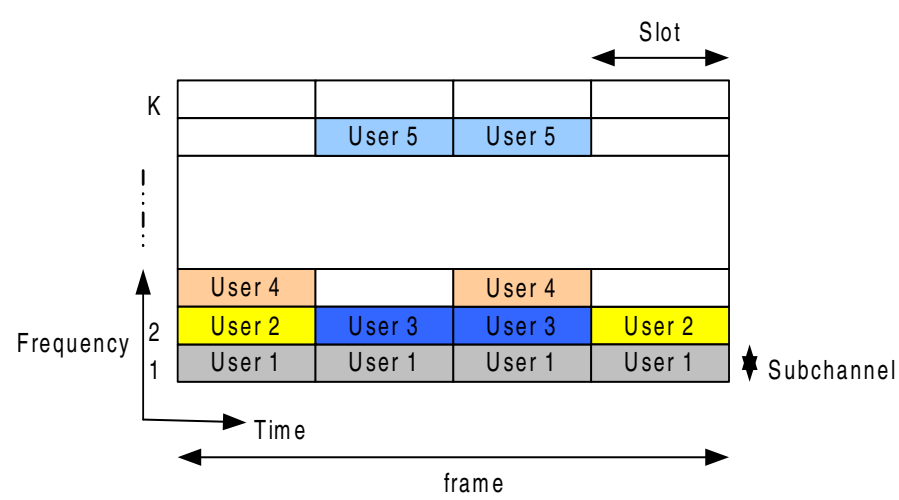

Fig. 1. Time-frequency resource allocation in OFDMA WiMax system

\section{B. AMC and cell decomposition}

In this work, and without loss of generality, we study AMC in the presence of path loss only which we characterize by a certain value $\xi$; high efficiency modulation is used for users where $\xi_{i} \ll \xi$, corresponding to a large $\mathrm{SNR}^{1}$. This results in the division of the cell into $r$ regions, $i=1 \ldots r$ (see Figure 2 ), which we assume to be concentric circles of radius $R_{i}$ for simplicity, but might be of different topology if we take into account other phenomena, such as fast-fading. In each region, users have the same modulation scheme and experience thus a corresponding bit rate which decreases as users get further from the base station.

To calculate the area covered by each modulation scheme, we must determine the maximal distance $R_{i}$ between Base Station (BS) and users using a corresponding modulation. This distance is determined using the maximal SNR a user should receive without data loss. Different values of received SNR for different modulation/coding schemes have been calculated in Reference [6] and are shown in Table I (first three columns). We shall now use them to calculate $R_{i}$.

${ }^{1}$ Please note that, for the time being, only the SNR matters, and not SINR, the Signal to Interference plus Noise ratio, as we now investigate the case of one cell in isolation. Later in the text, the multiple-cell setting will arise along with underlying interference and SINR.

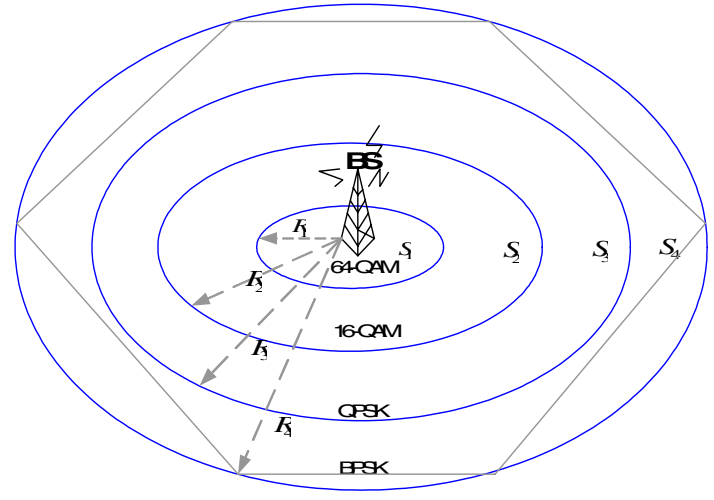

Fig. 2. Cell decomposition into regions

The path loss for the free space model is given by [8]:

$$
\begin{aligned}
P L_{i}[d B] & =-10 \log \left[G_{E} G_{R}\left(\frac{\lambda}{4 \pi R_{i}}\right)^{2}\right] \\
& =-10 \log G_{E}-10 \log G_{R}+20 \log \left(\frac{4 \pi R_{i}}{\lambda}\right)
\end{aligned}
$$

where $G_{E}$ is the emitter antenna gain, $G_{R}$ is the receiver antenna gain, $R_{i}$ is the distance between the emitter and the receiver and $\lambda$ is the wavelength. This path loss is also equal to:

$$
P L_{i}[d B]=P_{E}[d B m]-S N R[d B]-N[d B m]
$$

where $P_{E}$ is the emitted power and $N$ is the thermal noise (in units of decibels) which is equal to:

$$
N[d B m]=10 \log (\tau T W)
$$

$\tau=1.38 \cdot 10^{-23} \mathrm{watt} / K-H z$ is the Boltzmann constant, $T$ is the temperature in Kelvin $(T=290)$ and $W$ is the transmission bandwidth in $\mathrm{Hz}$.

Using the above equations, we can calculate the relationship between the distance and the SNR as follows:

$R_{i}=\frac{\lambda * 10^{\frac{P_{E}[d B m]+10 \log \left(G_{E}\right)[d B]+10 \log \left(G_{R}\right)[d B]-S N R[d B]-N[d B m]}{20}}}{4 \pi}$

The area of each region $S_{i}$ is given by:

$$
S_{i}=\pi \cdot\left(R_{i}^{2}-R_{i-1}^{2}\right)
$$

where $R_{0}=0$.

For the sake of illustration, let us consider the following example based on the licensed band for WiMAX to outdoor use in France which starts at a frequency of $3.4 \mathrm{GHz}$ and which has system bandwidth equal to $20 \mathrm{MHz}$. At this bandwidth, the thermal noise is equal to $-100.97 \mathrm{dBm}$. According to the maximum allowed Effective Isotropic Radiated Power (EIRP) of $1 W$, where the emitters are assumed to have an emission power $P_{E}$ of $1 W$ for users. We consider the case of antennas in $\mathrm{BS}$ and user equipment without gain. In Figure 3, we represent the distance assigned to SNR for switching points. The proportion of each surface area per PHY assumption is determined and shown in in Table I. 


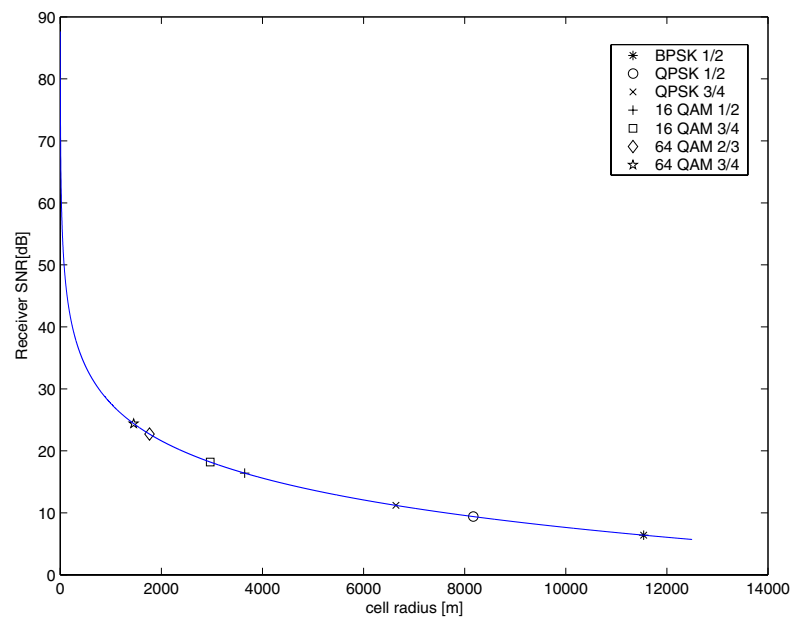

Fig. 3. Received SNR function of the distance

\begin{tabular}{|c|c|c|c|}
\hline Modulation & Coding rate & Receiver SNR(dB) & Surface [\%] \\
\hline BPSK & $1 / 2$ & 6.4 & 39.4 \\
\hline QPSK & $1 / 2$ & 9.4 & 20.75 \\
& $3 / 4$ & 11.2 & 28.0 \\
\hline 16 QAM & $1 / 2$ & 16.4 & 4.07 \\
& $3 / 4$ & 18.2 & 5.14 \\
\hline 64 QAM & $2 / 3$ & 22.7 & 0.9 \\
& $3 / 4$ & 24.4 & 1.74 \\
\hline
\end{tabular}

TABLE I

IEEE802.16 PHY ASSUMPTIONS

\section{Throughput of the cell}

The instantaneous physical bit rate $\bar{R}_{i}^{s, e}$ of streaming or elastic users in region $S_{i}$ is given by:

$$
\begin{aligned}
\bar{R}_{i}^{s, e} & =\frac{L_{i}^{s, e} \times K \times C \times \log _{2}(M)}{T_{s} \times S_{c}} \times(1-B L E R) \\
& =\quad L_{i}^{s, e} \times K \times B \times E_{i} \times(1-B L E R)
\end{aligned}
$$

where $L_{i}^{s, e}$ is the number of sub-channels to be assigned to streaming/elastic users in region $S_{i}, K$ is the number of data sub-carriers assigned to each sub-channel, $C$ is the coding rate of the $M$-ary modulation, $T_{s}$ is the OFDMA symbol duration given by:

$$
T_{s}=T_{b}+T_{g}
$$

with $T_{b}$ the useful symbol period (in units of microseconds) given by $\frac{N}{W \times n}$ and $T_{g}$ the guard period equal to $G \times T_{b}, W$ is the bandwidth $(M H z), n$ is the sampling factor, $G$ is the ratio of cyclic prefix (CP) to useful time, $S_{c}$ is the sector coefficient ( $S_{c}$ is equal to 1 in FUSC and 3 in PUSC 3 sectors), $B$ is the baud rate (symbols/sec), $E_{i}$ is the efficiency of the modulation (bits/symbol) in each region $S_{i}$ and $B L E R$ is the perceived Block Error Rate ${ }^{2}$.

Now, streaming flows have constant-bit rates, and so they are assigned a given number of sub-channels $L_{i}^{s}$ per region. As of (TCP-based) elastic calls, since they tolerate reduction in their throughput, they will simply share the left over capacity

\footnotetext{
${ }^{2}$ Note that for each value of SINR, we can determine a couple of values $(E, B L E R)$ and these values are determined by link level curves $E=$ $f(S I N R)$ and $B L E R=g(S I N R)$
}

on the basis of Processor Sharing (PS) [9]. The number of sub-channels $L_{i}^{e}$ allocated to an elastic call in region $S_{i}$ is thus given by:

$$
L_{i}^{e}=\left\lfloor\frac{L-\sum_{i=1}^{r} L_{i}^{s} n_{i}^{s}}{\sum_{i=1}^{r} n_{i}^{e}}\right\rfloor
$$

$\lfloor x\rfloor$ indicates the largest integer that is less than or equal to $x . n_{i}^{s}$ and $n_{i}^{e}$ are the number of streaming and elastic flows in region $S_{i}$.

\section{MARKOVIAN MODELING}

We assume that streaming calls arrive to region $S_{i}$ according to a Poisson process with intensity $\lambda_{i}^{s}$ and use $L_{i}^{s}$ subchannels for an exponentially distributed time with mean $1 / \mu^{s}$ independent of the share of the resources they get. We also assume that elastic flows arrive to the system according to a Poisson process with intensity $\lambda_{i}^{e}$ and assume for tractability that their service rate is exponentially distributed with mean $\mu_{i}^{e}=\frac{R_{i}^{e}}{E[Z]}$ where $E[Z]$ is the mean file size $^{3}$.

\section{A. Transition matrix}

Let us focus now on one cell. We can model our system as a Continuous Time Markov Chain (CTMC) by taking into account the proposed priorities for the integration of streaming and elastic flows as well as the way they share resources.

The state is characterized by the following row vector:

$$
\vec{n}:=\left(n_{1}^{s}, n_{2}^{s}, \ldots, n_{r}^{s}, n_{1}^{e}, n_{2}^{e}, \ldots, n_{r}^{e}\right)
$$

where $n_{i}^{s}$ and $n_{i}^{e}$, for $i=1 \ldots r$, represent the number of streaming and elastic calls in region $S_{i}$, respectively.

The state space of the system is given by:

$$
\mathfrak{S}:=\left\{\vec{n} \in \mathbb{N}^{2 \mathbf{r}} \mid \sum_{i=1}^{r}\left(L_{i}^{s} n_{i}^{s}+L_{i}^{e} n_{i}^{e}\right) \leq L\right\}
$$

where $L_{i}^{s}$ and $L_{i}^{e}$ denote the number of sub-channels allocated to streaming and elastic calls in region $S_{i}$ respectively and $L$ is the maximum number of sub-channels in the cell.

We now determine the steady-state probability vector $\vec{\Pi}=$ $\left\{\pi(\vec{n})_{\vec{n} \in \mathfrak{S}}\right\}$. Note that the corresponding system is non homogeneous as the departure rate of elastic calls depends on the overall number of calls in the system whereas streaming calls do not.

The solution of the steady-state distribution is obtained by solving the set of linearly independent equations given by:

$$
\begin{aligned}
& \vec{\Pi} \cdot Q=0 \\
& \sum_{\vec{n} \in \mathfrak{S}} \pi(\vec{n})=1
\end{aligned}
$$

To construct the transition matrix $Q$, we must consider all possible transitions between neighboring states. Let $q(\vec{n} \rightarrow$ $\left.\overrightarrow{n^{\prime}}\right)$ denote the transition probability from state $\vec{n}$ to neighboring states $\overrightarrow{n^{\prime}}$. Note that when we accept a new call in region $S_{i}, 1 \leq i \leq r$ the state is noted by $\vec{n}_{i^{+}}^{s, e}$ and when a call

${ }^{3}$ In fact, the total length of an elastic flow in units of packets is found to follow a log normal distribution, according to the measurement-based modelling [7] 
terminates the service the next state is $\vec{n}_{i^{-}}^{s, e}$. We thus have the following transition rates:

$$
\begin{aligned}
& q\left(\vec{n} \rightarrow \overrightarrow{n_{i^{+}}^{s}}\right)=\lambda_{i}^{s} \\
& q\left(\vec{n} \rightarrow \overrightarrow{n_{i^{-}}^{s}}\right)=n_{i}^{s} \mu^{s} \\
& q\left(\vec{n} \rightarrow \overrightarrow{n_{i^{+}}^{e}}\right)=\lambda_{i}^{e} \\
& q\left(\vec{n} \rightarrow \overrightarrow{n_{i^{-}}^{e}}\right)=\frac{n_{i}^{e} \mu_{i}^{e}(\vec{n})}{E[Z]}
\end{aligned}
$$

and the values $q(\vec{n} \rightarrow \vec{n})$ must be obtained as the sum of all terms in each line in matrix $\mathrm{Q}$ is equal to zero for $1 \leq i \leq r$.

\section{B. Performance measures}

Based on the steady-state probabilities of our CMTC, we now determine the performance measures relative to our model.

1) Blocking probability: The call blocking probabilities of both types of flows, $B_{i}^{s}$ and $B_{i}^{e}$ in region $S_{i}$, are obtained by summing up the steady state probability of saturation states:

$$
B_{i}^{s, e}=\sum_{\vec{n} \in \mathfrak{S}_{\mathfrak{i}}^{\mathfrak{s}, e}} \pi(\vec{n})
$$

where $\mathfrak{S}_{\mathfrak{i}}^{\mathfrak{s}, \mathfrak{e}}$ is the subset of states in $\mathfrak{S}$ for which any new call of class- $i$ is blocked when arriving to the system due to lack of resources. Formally,

$$
\mathfrak{S}_{\mathbf{i}}^{\mathbf{s}, \mathbf{e}}:=\left\{\vec{n} \in \mathfrak{S} \mid \sum_{k=1}^{r}\left(L_{k}^{s} n_{k}^{s}+L_{k}^{e} n_{k}^{e}\right)+l_{i}^{s, e}>L\right\}
$$

2) Mean transfer time: The mean transfer time of class- $i$ elastic flows can be calculated from the Little's formula by:

$$
\bar{T}_{i}=\frac{\bar{n}_{i}^{e}}{\lambda_{i}^{e}\left(1-B_{i}^{e}\right)}
$$

where $\overline{n_{i}^{e}}$ is the mean number of elastic flows in region $S_{i}$.

\section{REUSE PARTITIONING IN OFDMA WIMAX}

\section{A. Reuse partitioning}

Frequency reuse is the technique of increasing data capacity without compromising range. In the simplest case, the available channel is subdivided into groups, and the channel frequencies in each group are assigned to a cell with adjacent cells operating at different frequency sub-channels in order to maximize spectral efficiency. When a frequency reuse of one is used all cells operate on the whole frequency band. In a frequency reuse 3 scheme, each cell is allocated a third of the frequency band, and a 3-cell pattern is used. The first method suffers from the degradation in the quality of connection due to CoChannel Interference (CCI), while in the second method capacity is also reduced as a cell can only use a third of the total frequency band. To combat this, and to improve the spectral efficiency of cellular systems, a reuse partitioning scheme [4] can be applied. This means that a dynamical repartition of the bandwidth is applied between different interfering and non-interfering regions in the cell. In this section, we apply this reuse partitioning concept to OFDMA WiMAX (see Figure 4) and derive next the impact of this reuse partitioning on the number of collisions in the cell in avoiding interference at the cell edges.
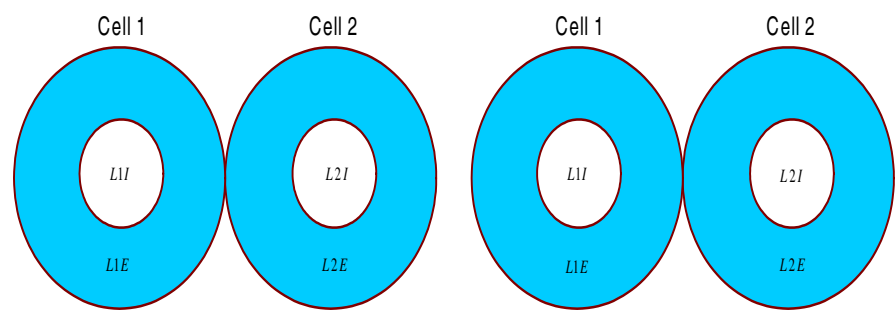

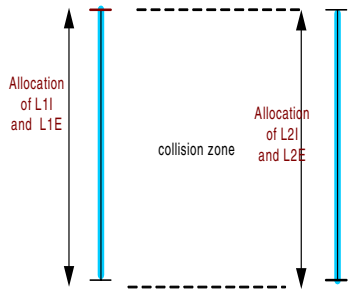

(a)

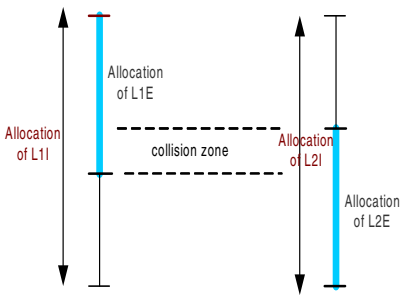

(b)
Fig. 4. Cell planning (a) without and (b) with frequency reuse partitioning

\section{B. Collisions}

There are several approaches as how to distribute available sub-carriers to sub-channels. The simplest one consists of picking randomly sub-carriers from available ones such that any available sub-carrier has the same probability to get allocated to an arriving user. We refer to this method as random allocation of sub-carriers which does not require any coordination between cells. Other allocation algorithms, such as based on frequency selectivity, are possible. We however restrict our focus in this work to random ones so as to highlight best the generic performance of OFDMA based systems. In the case of two cells, the following lemma establishes the mean number of collisions when the number of occupied sub-carriers is $(L \times K 1)$ and $(L \times K 2)$ in cell 1 and cell 2 respectively.

Lemma 1: The mean number of collisions in $L$ subchannels is given by:

$$
E[C \mid K 1, K 2]=L \cdot E^{\prime}[C \mid K 1, K 2]
$$

where $E^{\prime}[C \mid K 1, K 2]$ is the mean number of collisions in one group of sub-carriers and is in turn given by:

$$
E^{\prime}[C \mid K 1, K 2]=\sum_{c=c_{\min }}^{c_{\max }} c \cdot \operatorname{Pr}(c \mid K 1, K 2)
$$

$\operatorname{Pr}(c \mid K 1, K 2)$ being the probability of having $c$ collisions in this group and is equal to:

$$
\frac{\sum_{n 1 c=0}^{K 1-c}\left(\begin{array}{c}
N / L \\
c
\end{array}\right)\left(\begin{array}{c}
N / L-c \\
n 1 c
\end{array}\right)\left(\begin{array}{c}
N / L-c-n 1 c \\
K 2-c
\end{array}\right)}{\sum_{c=c_{\text {min }}}^{c_{\max }} \sum_{n 1 c=0}^{K 1-c}\left(\begin{array}{c}
N / L \\
c
\end{array}\right)\left(\begin{array}{c}
N / L-c \\
n 1 c
\end{array}\right)\left(\begin{array}{c}
N / L-c-n 1 c \\
K 2-c
\end{array}\right)}
$$

$c$ is the number of colliding sub-carriers. It belongs to $\left\{c_{\min }: c_{\max }\right\}$ with

$$
c_{\text {min }}=\max (0, K 1+K 2-N / L)
$$

and

$$
c_{\max }=\min (K 1, K 2, N / L)
$$

and $n 1 c$ is the number of non-colliding sub-carriers in cell 1 ; $n 1 c \in\{0: K 1-c\}$. 
Proof: Let $K_{i}$ denote the number of occupied subcarriers in each group of $N / L$ sub-carriers in cell $i$. Recall that $c$ is the number of collisions in each group; it ranges between above-mentioned $c_{\min }$ and $c_{\max }$.

The probability of having $c$ collisions in this group of subcarriers is equal to the ratio between the number of possible allocations such that the number of colliding sub-carriers is $c$ to the total number of possible allocations.

Collisions are possible in the group of $(N / L)$ sub-carriers, which implies that we have $\left(\begin{array}{c}N / L \\ c\end{array}\right)$ possible combinations for collisions in the collision zone of cell 1 and $\left(\begin{array}{c}N / L-c \\ n 1 c\end{array}\right)$ possible combinations for non-colliding sub-carriers in the same cell. Knowing that cell 1 has chosen a given set of $K 1$ sub-carriers, the number of possible combinations for the non-colliding subcarriers in cell 2 is equal to: $\left(\begin{array}{c}N / L-c-n 1 c \\ K 2-c\end{array}\right)$. The number of possible allocations such that we have $c$ colliding sub-carriers is then the product of $\left(\begin{array}{c}N / L \\ c\end{array}\right)\left(\begin{array}{c}N / L-c \\ n 1 c\end{array}\right)\left(\begin{array}{c}N / L-c-n 1 c \\ K 2-c\end{array}\right)$ over all possible combinations of non-colliding sub-carriers.

Now, the total number of possible allocations is then the summation over all possible combinations : $\sum_{c=c_{\min }}^{c_{\max }}\left(\begin{array}{c}N / L \\ c\end{array}\right)\left(\begin{array}{c}N / L-c \\ n 1 c\end{array}\right)\left(\begin{array}{c}N / L-c-n 1 c \\ K 2-c\end{array}\right)$.

The probability of having $c$ collisions is then given by Eqn. (14). The expected number of collisions in one group is then obtained by Eqn. (13). Based on the collisions in each of the $L$ groups, the total number of collisions $C$ in the total channel (bandwidth) is given by: $C=\sum_{i=1}^{L} c_{i}$. These collisions being independent and the groups identical, we obtain the mean number of collisions in Eqn. (12).

Lemma 2: The mean number of collisions in the system is equal to [11]:

$$
E[C]=\sum_{K 1, K 2}\left(\prod_{i=1}^{2} \Pi_{i}\left(K_{i}\right)\right) E[C \mid K 1, K 2]
$$

where $E[C \mid K 1, K 2]$ is given from Equation (12) and $\Pi_{i}\left(K_{i}\right)=\sum_{\overrightarrow{n_{i}} \in \mathfrak{S}} \pi\left(\overrightarrow{n_{i}}\right) \operatorname{Pr}\left(K_{i} \mid \overrightarrow{n_{i}}\right)$ is the probability of having $L K_{i}$ sub-carriers in cell $i$.

\section{NUMERICAL RESULTS}

We now present some numerical results obtained by the simulation of our flow model when applied to an OFDMA system with an FFT size of 1024 sub-carriers and considering without loss of generality 2 regions with AMC respectively 16-QAM $3 / 4\left(E_{1}=3 \mathrm{bit} / \mathrm{symbol}\right)$ and QPSK $1 / 2\left(E_{2}=1 \mathrm{bits} / \mathrm{symbol}\right)$. These parameters of efficiency corresponds to the transmission modes with convolutionally coded modulation [12].

Moreover, let $L=5 ; L_{1}^{s}=L_{2}^{s}=1 ; \lambda_{i}^{s}=0.01 ; \lambda_{i}^{e}=0.01$; $\mu^{s}=0.02 ; B L E R=0 ; E[Z]=500 K b$.

Based on Equation (5) and using a baud value $B=$ $2666 \mathrm{symbol} / \mathrm{sec}$ and $K=48, R_{1}^{s}=128 \mathrm{Kbps}, R_{2}^{s}=$ 384 Kbps.

We first start with the case of a single cell where the number of sub-carriers is the same in the outer and inner regions, i.e., without reuse partitioning.

Figures 5 and 6 show the blocking probability for, respectively, streaming and elastic flows as a function of an increasing arrival rate of streaming flows. And this for the two

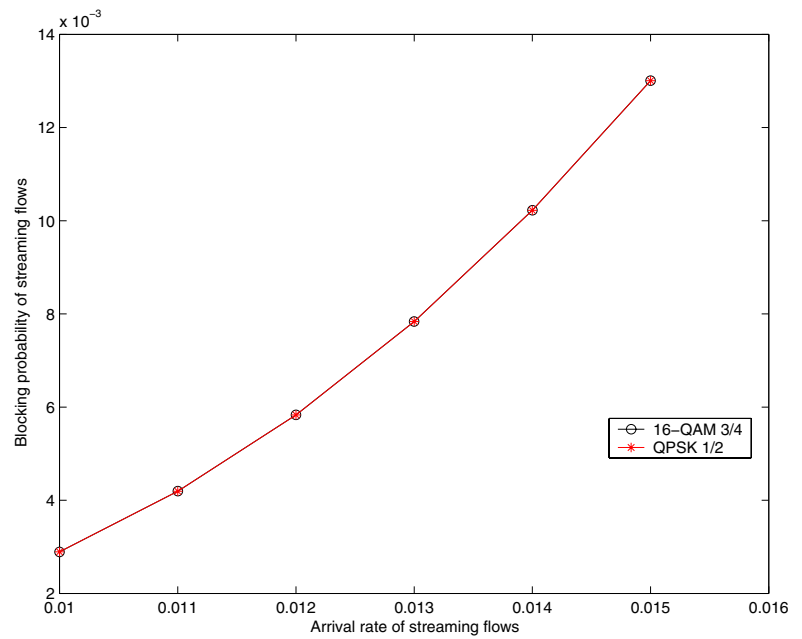

Fig. 5. Blocking probability for streaming flows in two regions

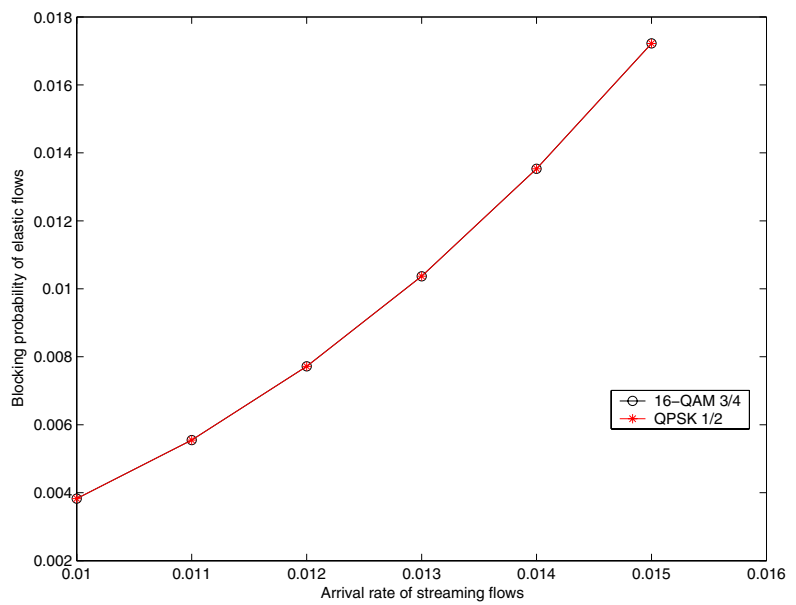

Fig. 6. Blocking probability for elastic flows in two regions

regions, the inner one (labeled 16-QAM 3/4) and the outer one (labeled QPSK 1/2).

We observe in terms of blocking that we have only one class for streaming flows in the inner and outer region. Data flows however are elastic and share capacity among themselves in a fair manner on the basis of processor sharing. This makes them obtain the same blocking rate. They however obtain different mean transfer times in each region corresponding to the bit rate they achieve therein, as shown in Figure 7.

We next turn to the case when reuse partitioning is enabled. With respect to Figure 4, the number of sub-channels in the inner region $L 1 I$ remains unchanged whereas their number in the outer region is decreased, $L 1 E=4$.

This decrease in the number of sub-channels in the outer region leads to new blocking probabilities for both types of traffic as shown in Figures 8 and 9 for, respectively, streaming and elastic flows as a function of an increasing arrival rate of streaming flows for the two regions, inner and outer.

For streaming flows, the blocking probability in the inner region decreases as flows in this region have now access to all sub-channels whereas flows in the outer region do not. The latter have thus higher blocking. For data flows however, both 


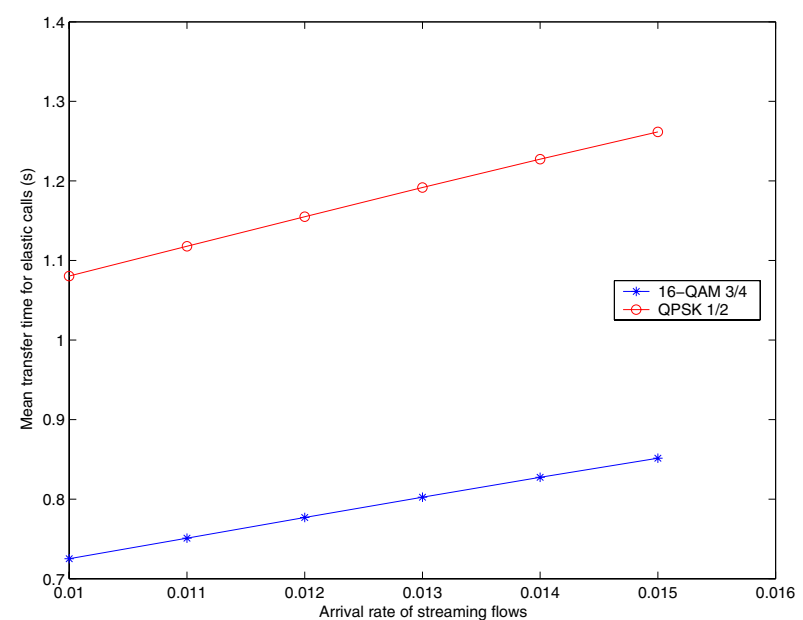

Fig. 7. Mean transfer time for elastic flows in two regions

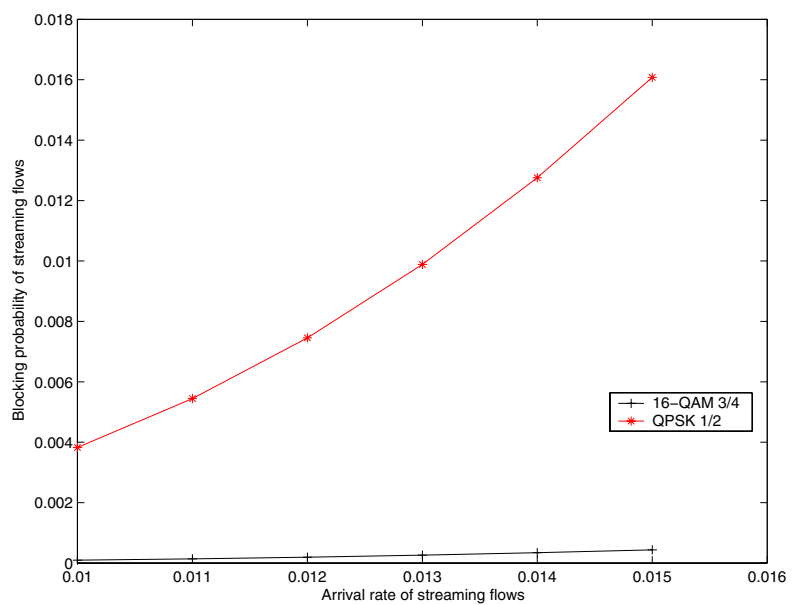

Fig. 8. Blocking probability for streaming flows with reuse partitioning

blocking rates, inner and outer, increase with respect to the case with no frequency reuse, as more streaming flows are now accepted; with a higher increase in the outer ring as less sub-channels are now available.

Now, the mean number of collisions in the system is equal to $10^{-2}$ without reuse partitioning and half that when reuse partitioning is enabled. And so, one would expect a higher overall throughput for the cell. This is the case, as shown in Figure 10, and which means that the decrease in the number of collisions when reuse partitioning is enabled improves the overall throughput.

\section{CONCLUSION}

In this paper, we considered the calculation of capacity of the downlink of OFDMA-based IEEE802.16 WiMAX systems, focusing on the effect of AMC as well as reuse partitioning and underlying collisions in the case of more than one cell. And this, for two types of flows, constant-bit-rate streaming and elastic data.

Our next step shall be on QoS issues, especially for streaming traffic which suffers from degraded performance as users get away from the base station in the case of mobility.

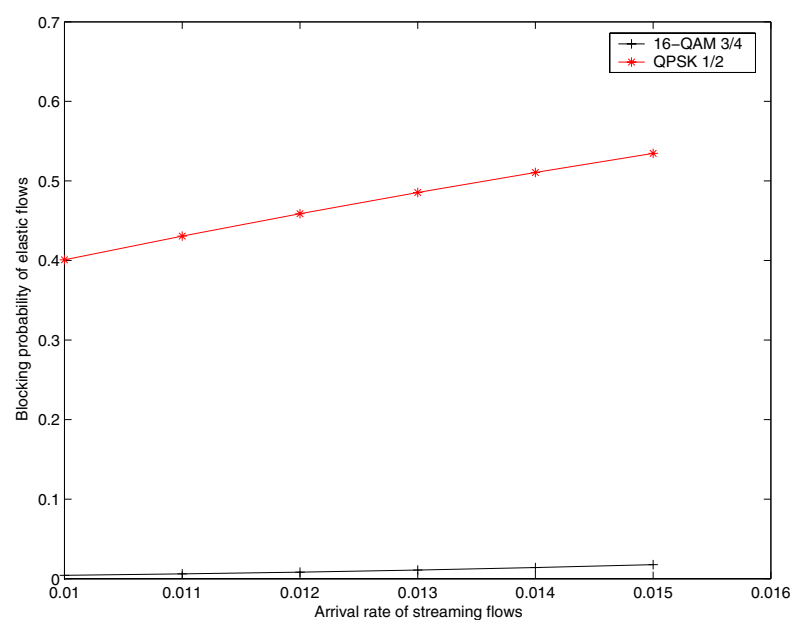

Fig. 9. Blocking probability for elastic flows with reuse partitioning

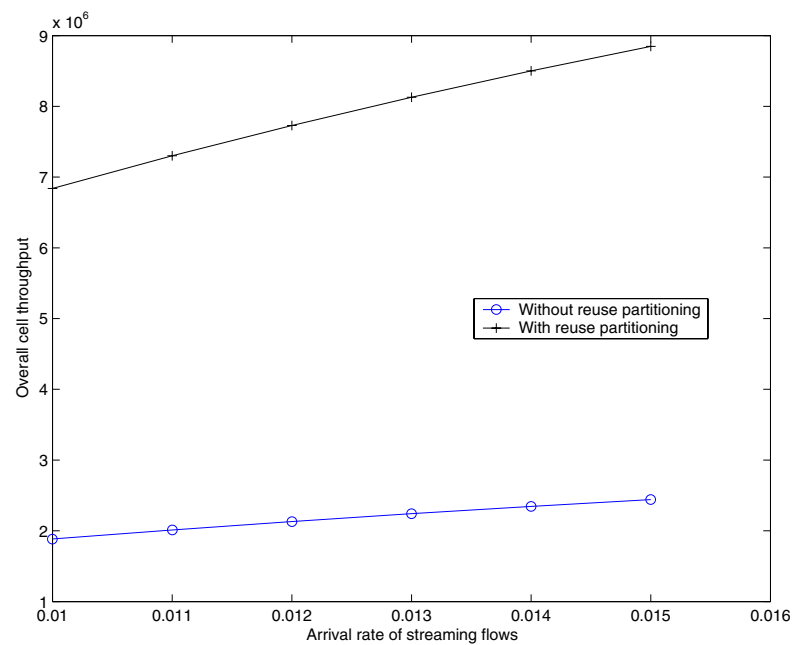

Fig. 10. Overall cell throughput

\section{REFERENCES}

[1] www.wimaxforum.org.

[2] A. Molisch, Wireless Communications, IEEE Press, 2005.

[3] H. Yaghoobi, Scalalable OFDMA Physical Layer in IEEE 802.16 WirelessMAN, Intel Technology Journal, pp. 201-212, August 2004.

[4] M.Johansson, Dynamic Reuse Partitioning Within Cells Based on Local Channel and Arrival Rate Fluctuations, Technical Report, Uppsala University, Sweden, 2005. http://www.signal.uu.se/Staff/mj/pub/intercell.pdf

[5] IEEE 802.16-2005, Part 16: Air Interface for Fixed and Mobile Broadband Wireless Access Systems, IEEE Standard for local and Metropolitan Area Networks, February 2006.

[6] IEEE 802.16-2004, Part 16: Air Interface for Fixed Broadband Wireless Access Systems, IEEE Standard for local and Metropolitan Area Networks, October 2004.

[7] A. B. Downey, The structural cause of file size distributions, ACM SIGMETRICS Performance Eval. Rev., vol. 29, pp. 328329, June 2001.

[8] G. L. Stuber, Principles of Mobile Communication, 2nd ed. Norwell, MA:Kluwer, 2001.

[9] N. Benameur, S. Ben Fredj, F. Delcoigne, S. Oueslati-Boulahia and J.W. Roberts, Integrated Admission Control for Streaming and Elastic Traffic, QofIS 2001, Coimbra, September 2001.

[10] C. Tarhini, T. Chahed, System capacity in OFDMA-based WiMAX, ICSNC 2006, Tahiti, November 2006.

[11] S-E. Elayoubi, B. Fourestie and X. Auffret, On the capacity of OFDMA 802.16 systems, ICC 2006, Istanbul, June 2006.

[12] Q.Liu, S.Zhou, G.B.Giannakis, Queuing with Adaptive Modulation and Coding over wireless links: Cross-Layer analysis and design, IEEE transactions on wireless communications, vol.4, NO.3, May 2005. 